\title{
Aplicación de técnicas cinéticas no isotérmicas en la reducción de tres minerales de hierro de grado comercial
}

\author{
E. Bedolla $\left.{ }^{*}\right)$, C.A. León $(*)$ y E.A. Aguilar $\left.{ }^{*}\right)$ \\ Resumen Se estudió el mecanismo de reducción en aglomerados de mineral de hierro mediante análisis termo- \\ gravimétricos tanto isotérmicos como no isotérmicos, complementando el estudio por caracterización \\ estructural de muestras parcial y completamente reducidas. Se estudiaron tres tipos de pelets hematí- \\ ticos grado comercial. Los mecanismos de reducción se determinaron isotérmicamente y resultaron \\ ser modelos de control por reacción química. La reducción no isotérmica se realizó en un TGA \\ CAHN-171 de 600 a $1.000{ }^{\circ} \mathrm{C}$ a velocidad de calentamiento lineal de 4,7 y $10^{\circ} \mathrm{C} / \mathrm{min}$, en atmósferas \\ reductoras de $\mathrm{H}_{2}(100 \%)$ y $\mathrm{H}_{2}-\mathrm{CO}(95: 5)$. Los parámetros cinéticos se evaluaron por las técnicas \\ propuestas por Coats \& Redfern, Dixit \& Ray y Prakash \& Ray. Se encontró que a menor velocidad \\ de calentamiento, mayor es el grado de reducción no isotérmico y mayor la energía de activación del \\ proceso. La energía de activación con la mezcla $\mathrm{H}_{2}-\mathrm{CO}$, siempre fue mayor que con $\mathrm{H}_{2}$ puro.
}

Palabras clave: Reducción no isotérmica. Cinética de reducción. Mineral de hierro. Energía de activación. Velocidad de calentamiento.

\section{Application of nonisothermal kinetic techniques on the reduction of three commercial iron ore agglomerates.}

\begin{abstract}
The mechanism of reduction of iron ore agglomerates by both isothermal and nonisothermal TG studies was investigated, and the work was complemented with the structural characterization of the total and partially reacted samples. Three different commercial hematite pellets were studied. The mechanisms of reduction were obtained under isothermal conditions, resulting in a fitting to chemical reaction models. Nonisothermal reduction was carried out using a TGA system (CAHN TG-171) from 600 to $1,000{ }^{\circ} \mathrm{C}$ maintaining a lineal heating rate of 4,7 and $10{ }^{\circ} \mathrm{C} / \mathrm{min}$, and the reducing atmospheres used were $\mathrm{H}_{2}(100 \%)$ and $\mathrm{H}_{2}-\mathrm{CO}(95: 5)$. The kinetic parameters were evaluated by Coats \& Redfern, Dixit \& Ray and Prakash \& Ray techniques. It was found that the lower the heating rate, the higher the reduction degree and the higher activation energy. The activation energy for reduction with the mixture $\mathrm{H}_{2}-\mathrm{CO}$ was always higher than that obtained with pure $\mathrm{H}_{2}$.
\end{abstract}

Keywords: Nonisothermal reduction. Reduction kinetics. Iron ore. Activation energy. Heating rate.

\section{INTRODUCCIÓN}

Diversos autores han investigado el uso de datos termogravimétricos para evaluar los parámetros cinéticos de reacciones en fase sólido-gas que envuelvan pérdida o ganancia en peso, lo que ha llevado a numerosas publicaciones sobre la cinética de reducción y la formulación de modelos matemáticos para la interpretación de datos cinéticos.

La mayoría de las aportaciones al estudio no isotérmico corresponden principalmente al desarrollo

(•) Trabajo recibido el día 27 de agosto de 1996.

(*) Instituto de Investigaciones Metalúrgicas. Universidad Michoacana de San Nicolás de Hidalgo. Apdo. postal 52-B. Morelia, Mich. (México) teórico de métodos diferenciales e integrales para la evaluación de los parámetros cinéticos, lo que incluye mecanismos de reacción, energías de activación del proceso, etc. (1-8). Sin embargo, existe poca información en la bibliografía donde se estudie el efecto de las variables atmósfera reductora, velocidad de calentamiento, composición de solidos y gases, tamaño de las materias primas, etc., sobre el grado de reducibilidad, parámetros cinéticos y cambios microestructurales que se presentan durante el proceso de reducción no isotérmico.

Considerando que la gran mayoría de los sistemas actuales se desarrollan en condiciones no isotérmicas, en el presente trabajo se lleva a cabo un intento para entender el mecanismo de reducción no isotérmico de pelets comerciales de mineral de 
hierro realizando estudios termogravimétricos. También se estudia el efecto de la velocidad de calentamiento y la atmósfera reductora en los parámetros cinéticos resultantes. El estudio cinético se complementó con diferentes técnicas de caracterización y se determinó la relación entre los cambios morfológicos y estructurales con los cambios en las variables de operación y los parámetros cinéticos.

La reducción gaseosa de la fase hematites por gases se compone de una serie de procesos individuales, donde se asume que la etapa más lenta de la secuencia es el factor controlante de la velocidad. Existen 19 posibles modelos cinéticos propuestos en la literatura que pertenecen a reacciones sólidogas (9). La reducción de la wustita a hierro es cinéticamente la etapa más importante del proceso global de reducción, pero las etapas iniciales no dejan de ser importantes, porque las condiciones a las que se forman la magnetita y la wustita afectan a la estructura y a la velocidad de formación del hierro.

La porosidad de los óxidos de hierro es importante para determinar el área superficial de la que dispone el sólido para la reducción y, por tanto, afecta a la velocidad de reacción química y al correspondiente mecanismo de reacción. El-Geassy y Nasr (10) encontraron que el incremento en la porosidad de pastillas de mineral de hierro disminuye la energía de activación en la reducción con $\mathrm{H}_{2}$ puro.

Towhidi y Szekely (11) encontraron que un mayor contenido de $\mathrm{H}_{2}$ en las mezclas reductoras, hace que se incremente notablemente la velocidad de reducción de los pelets hematíticos grado comercial cuando se reducen con mezclas $\mathrm{H}_{2}-\mathrm{CO}$. Sin embargo, la reducción de los óxidos de hierro con $\mathrm{H}_{2}$ puro origina la formación de capas densas de hierro metálico que envuelven la fase wustita. Se ha demostrado que la adición de pequeñas cantidades de monóxido de carbono en mezclas de $\mathrm{H}_{2}$ incrementa la reducción en las etapas finales del proceso. Algunos autores han propuesto mecanismos para demostrar cómo mediante la adición de $\mathrm{CO}$ se mejora el proceso de reducción (12 y 13).

En la bibliografía se proponen diferentes métodos para el tratamiento de datos cinéticos no isotérmicos (3, 8 y 14-16). Las técnicas usadas en el presente trabajo son las propuestas por Coats \& Redfern (1), Dixit \& Ray (2) y Prakash \& Ray (3).

\subsection{Evaluación de los parámetros cinéticos}

Los métodos para el análisis de datos cinéticos no isotérmicos establecen tres consideraciones: a) comportamiento isocinético de la reacción, b) conocimiento del modelo cinético de reacción a partir de datos isotérmicos para el mismo sistema, y c) el mecanismo encontrado es válido en el rango de temperaturas establecido isotérmicamente.

\subsubsection{Método de Coats \& Redfern}

Se trata de un método integral empleado cuando la velocidad de calentamiento es constante. La expresión final esta dada por:

$$
\ln \left[\frac{g(\alpha)}{T^{2}}\right]=\ln \left[\frac{A R}{a E}\left(1-\frac{2 R T}{E}\right)\right]-\frac{E}{R T}
$$

\subsubsection{Método de Dixit \& Ray}

Se aplica en experimentos donde la velocidad de calentamiento se da por incremento constante de la temperatura.

$$
\ln g(\alpha)=\ln \left(\frac{A R}{b E}\right)-\frac{E}{R T}
$$

\subsubsection{Método de Prakash \& Ray}

Es una técnica de análisis diferencial que puede aplicarse para condiciones fluctuantes de temperatura.

$$
\ln \left[\frac{d \alpha / d T) f^{\prime}{ }_{1}(t)}{f(\alpha)}\right]=\ln A-\frac{E}{R T}
$$

donde, $\alpha$ es la fracción reducida, $g(\alpha)=k t$ es la forma integral de la ecuación cinética y $f(\alpha)$ representa un modelo hipotético del mecanismo de reacción igual a $l / g^{\prime}(\alpha)$. $T$ es la temperatura $(\mathrm{K}), t$ el tiempo de reacción (min), $E$ la energía de activación (kJ/mol), $A$ es el factor pre-exponencial, y $R$ la constante universal de los gases $(\mathrm{kJ} / \mathrm{mol} \mathrm{K})$. La variable $a$ es una expresión de la velocidad de calentamiento lineal igual a $d T / d t(\mathrm{~K} / \mathrm{min}), \mathrm{y} f^{\prime}{ }_{1}(t)$ es la primera derivada de la función que presenta la variación de la temperatura con el tiempo $T=f^{l}(t)$. Al representar gráficamente el término de la izquierda de cada ecuación frente al inverso de la temperatura se obtiene una línea recta, cuya pendiente es igual a $-E / R$.

El objetivo del presente diseño experimental es estudiar el comportamiento cinético de pelets de mineral de hierro de grado comercial cuando se someten a reducción no isotérmica a diferentes velocidades de calentamiento y agentes reductores. Para caracterizar muestras reducidas y sin reducir en diferentes etapas de los experimentos, se utilizaron técnicas de microscopía óptica, medición de área superficial por BET, difracción de rayos-X, microscopía electrónica de barrido y microsonda. 


\section{PROCEDIMIENTO EXPERIMENTAL}

\subsection{Materias primas}

En el presente trabajo se emplearon tres tipos de pelets de mineral de hierro de grado comercial usados en la producción de esponja de hierro en la empresa siderúrgica IMEXA S.A. de Michoacán (México). Estos pelets proceden de Peña Colorada (México), SIDOR (Venezuela) y CVDR (Brasil), cuyo análisis se muestra en la tabla I. Como agentes reductores se emplearon las mezclas de gases $\mathrm{H}_{2}$ (100\%) y $\mathrm{H}_{2}-\mathrm{CO}(95: 5)$.

\subsection{Condiciones experimentales}

\subsubsection{Estudio isotérmico}

En un analizador termogravimétrico, CAHN TGA-171, con tubo de reacción de alúmina de $3 \mathrm{~cm}$ de diámetro interno y en el rango de temperaturas $600-1.000{ }^{\circ} \mathrm{C}$, se redujeron isotérmicamente pelets de $4,5 \mathrm{~g}$. El calentamiento y enfriamiento de las muestras se realizó en atmósfera inerte. El flujo de gas reductor se mantuvo constante e igual a 180 $\mathrm{ml} / \mathrm{min}$ para evitar la formación de la capa límite, de acuerdo con las especificaciones de diseño del equipo experimental empleado.

\subsubsection{Estudio en condiciones no isotérmicas}

En el contenedor de muestras se colocaron dos pelets con un peso total de aproximadamente $9 \mathrm{~g}$, que se sometieron a diferente velocidad de calentamiento desde temperaturas bajas. Cuando se alcanzó la temperatura de $300{ }^{\circ} \mathrm{C}$ se permitió el acceso del gas reductor hasta llegar a la temperatura final de $1.000{ }^{\circ} \mathrm{C}$. La reducción no isotérmica se practicó a tres velocidades de calentamiento lineal de 4, 7 y $10{ }^{\circ} \mathrm{C} / \mathrm{min}$. El calentamiento previo a la entrada del gas reductor y el enfriamiento una vez terminado el ciclo de calentamiento, se realizaron en atmósfera inerte de gas nitrógeno.

\section{RESULTADOS}

Para el estudio del mecanismo de reacción es necesario interpretar la pérdida de peso de las curvas de reducción en función de la fracción reducida. El estudio termogravimétrico es una técnica que se fundamenta en la determinación continua del cambio de peso de las muestras tratadas. Para el caso de la reducción del mineral de hierro, este cambio representa la pérdida de peso asociada con la remoción completa de oxígeno ligado al hierro, donde el grado de reducción, se calcula por la fórmula:

$$
\text { Grado de reducción }=\alpha=\frac{\Delta W}{W}
$$

donde:

$\Delta W=$ peso de la muestra antes de la reducción menos el peso de la muestra después de la reducción $(\mathrm{g})$

$W=$ pérdida de peso posible correspondiente a $100 \%$ de reducción $(\mathrm{g})$.

\subsection{Reducción en condiciones isotérmicas}

La importancia del desarrollo isotérmico en el estudio de la cinética de reducción no isotérmica se fundamenta en la determinación del mecanismo cinético controlante de la reacción, el cual será empleado en el estudio cinético no isotérmico de

TABLA I.- Características químicas y físicas del mineral de hierro utilizado

TABLE I.-Chemical and physical characteristics of iron ore used

\begin{tabular}{|c|ccccccc|}
\hline Material & $\mathrm{Fe}^{\mathrm{T}}$ & $\mathrm{Fe}^{2+}$ & $\mathrm{SiO}_{2}$ & $\mathrm{CaO}$ & $\mathrm{MgO}$ & $\mathrm{Al}_{2} \mathrm{O}_{3}$ & $\mathrm{~S}$ \\
\hline Peña C & 65,80 & 0,35 & 2,36 & 1,95 & 0,51 & 1,06 & 0,012 \\
SIDOR & 65,80 & 0,41 & 2,35 & 2,14 & 0,48 & 0,78 & 0,004 \\
CVDR & 68,02 & 0,22 & 1,13 & 0,64 & 0,29 & 0,48 & 0,003 \\
\hline & & & Peña C. & SIDOR & $\mathrm{CVDR}$ \\
\cline { 3 - 6 } & & 2,25 & 2,06 & 2,10 \\
Densidad, $\mathrm{g} / \mathrm{cm}^{3}$ & & 24,4 & 24,8 & 24,2 \\
Porosidad, \% & & 0,072 & 0,097 & 0,109 \\
Área superficial, $\mathrm{m}^{2} / \mathrm{g}$ & & 9 & 9 & 9 \\
Diámetro promedio, $\mathrm{mm}$ & & & & & \\
\hline
\end{tabular}


acuerdo con los fundamentos establecidos para el uso de estas técnicas.

En esta etapa experimental se confirma que la reducción de los pelets hematíticos produce esponja de hierro porosa, cuya estructura depende de la temperatura de reducción, y se comprueba, además, que la densificación del hierro metálico por efectos de sinterización es altamente sensible a la temperatura. Para los diferentes tipos de pelets, se observó que el incremento de la temperatura favorece la velocidad de reacción e incrementa el grado de reducción, disminuyendo la cantidad de óxidos presentes y también la porosidad total de la muestra debido a la sinterización de los granos de hierro. En la reducción con ambas mezclas, la fracción reducida se aproximó a la unidad con el incremento de la temperatura.

\subsection{Determinación del mecanismo de reacción}

Para identificar el modelo cinético seguido por el proceso de reducción, se usó la técnica de las curvas de reducción normalizadas en el tiempo (reduced-time plots), donde los datos experimentales se comparan directamente con curvas teóricas estándar.

La obtención de las curvas estándar normalizadas de reducción se fundamenta en la siguiente expresión cinética:

$$
g(\alpha)=k t
$$

donde $g(\alpha)$ es una función apropiada de $\alpha$ para los diferentes modelos cinéticos.

Si $t_{0,5}$ es el tiempo necesario para obtener $0,5 \mathrm{de}$ fracción reaccionada $(\alpha=0,5)$, se tiene que:

$$
g(\alpha)_{\alpha=0,5}=k t_{0,5}
$$

El coeficiente de velocidad $k$ es constante para una temperatura dada, por tanto, al dividir la ecuación [5] entre la ecuación [6] se obtiene la relación cinética:

$$
g(\alpha)=g(\alpha)_{\alpha 0,5}\left(\frac{t}{t_{0,5}}\right)
$$

La ecuación [7] es adimensional e independiente de la constante de velocidad de reacción. Se tiene que para todo mecanismo de reacción en particular, una simple ecuación de este tipo representa todos los datos cinéticos, independientemente de la naturaleza del sistema, temperatura u otros factores que afecten a la velocidad de reacción. A cada mecanismo le corresponde una sola curva patrón. El uso de estos gráficos ha sido previamente citado en la bibliografía (17 y 18). En el presente estudio se empleó el valor $t_{0,5}$, ya que para todas las condiciones experimentales, el grado de reducción, $\alpha$, resultó mayor que 0,5 .

Las curvas normalizadas estándar de los diferentes mecanismos se construyen al representar graficamente los valores del grado de reducción, $\alpha$, en función de los valores $t / t_{0,5}$ calculados por la ecuación [7].

Las curvas normalizadas que resultan de los datos experimentales, se superponen a las curvas teóricas para determinar el mecanismo controlante de la reacción. Como el estudio no isotérmico considera comportamiento isocinético de la reacción, se elige el modelo que, en promedio, presente el mejor ajuste para todo el rango de temperatura estudiado. Una vez determinados mediante esta técnica, los modelos cinéticos fueron validados comprobando su linealidad por gráficos $g(\alpha)$ frente a $t$.

La tabla II presenta resumidos los mecanismos de reacción obtenidos para las diferentes condiciones experimentales. Los mecanismos resultantes corresponden a procesos controlados por reacción química, cuya expresión matemática difiere por el exponente al cual se encuentra elevada la base (4 y 19):

$$
\begin{gathered}
\text { Modelo cinético de simetría cilíndrica (CG2) } \\
1-(1-\alpha)^{1 / 2}=k t \\
\text { Modelo cinético de simetría esférica (CG3) } \\
1-(1-\alpha)^{1 / 3}=k t
\end{gathered}
$$

\subsection{Reducción en condiciones no isotérmicas}

En la reducción no isotérmica, la cantidad de flujo de calor está siempre cambiando y no se guarda un equilibrio termodinámico constante en función de la temperatura, por lo que, teóricamente, es posible que el mecanismo no esté controlado por un régimen simple. Sin embargo, de acuerdo con los principios establecidos en la bibliografía para el tratamiento de datos cinéticos no isotérmicos, se ha

TABLA II.-Mecanismos de control determinados en las diferentes condiciones experimentales

TABLE II.-Mechanisms found at the different experimental conditions

\begin{tabular}{|c|c|c|c|}
\hline Gas reductor & Peña C. & SIDOR & CVDR \\
\hline $\mathrm{H}_{2}(100 \%)$ & $\mathrm{CG} 2$ & $\mathrm{CG} 3$ & $\mathrm{CG} 2$ \\
$\mathrm{H}_{2}-\mathrm{CO}(95: 5)$ & $\mathrm{CG} 3$ & $\mathrm{CG} 3$ & $\mathrm{CG} 2$ \\
\hline
\end{tabular}

CG2: Modelo de simetría cilíndrica 1- $(1-\alpha)^{1 / 2}$ (Control por reacción química).

CG3: Modelo cinético de simetría esférica 1- $(1-\alpha)^{1 / 3}$ (Control por reacción química). 
considerado un solo mecanismo controlante de la reacción para cada caso experimental.

En un ciclo de calentamiento no isotérmico, la temperatura aumenta conforme transcurre el tiempo, induciendo un aumento en el grado de reducción. En el caso de un proceso isotérmico, las curvas de reducción presentan diferencias en la pendiente al inicio y al final del proceso, mientras que en la reducción no isotérmica los cambios son menores y son más evidentes cuando los experimentos se realizan a baja velocidad de calentamiento (Fig. 1). Estos cambios en la pendiente pueden indicar reacciones secuenciales que se presenten en el proceso.

La figura 2 presenta micrografías obtenidas por microscopía electrónica de barrido donde se observa la morfología de muestras parcialmente reducidas con $\mathrm{H}_{2}$-CO a 2,7 y $18 \%$. Se observa que al inicio de la reducción, los granos de óxido, que en su mayoría son magnetita y parte hematites, presentan formas definidas con caras planas y formación de bordes angulares entre ellas (Fig. 2a). Con el transcurso de la reducción hasta $18 \%$ y temperatura aproximada de $650{ }^{\circ} \mathrm{C}$, aparece la fase wustita y la morfología de los granos cambia por efecto de la temperatura. Las partículas pierden su forma original y se produce la esferoidización de los granos (Fig. 2b).

\subsubsection{Efecto de la velocidad de calentamiento}

La temperatura a la cual se manifestó el inicio de la reducción en condiciones no isotérmicas fue en el rang de 450 a $480^{\circ} \mathrm{C}$ para todos los casos. Durante

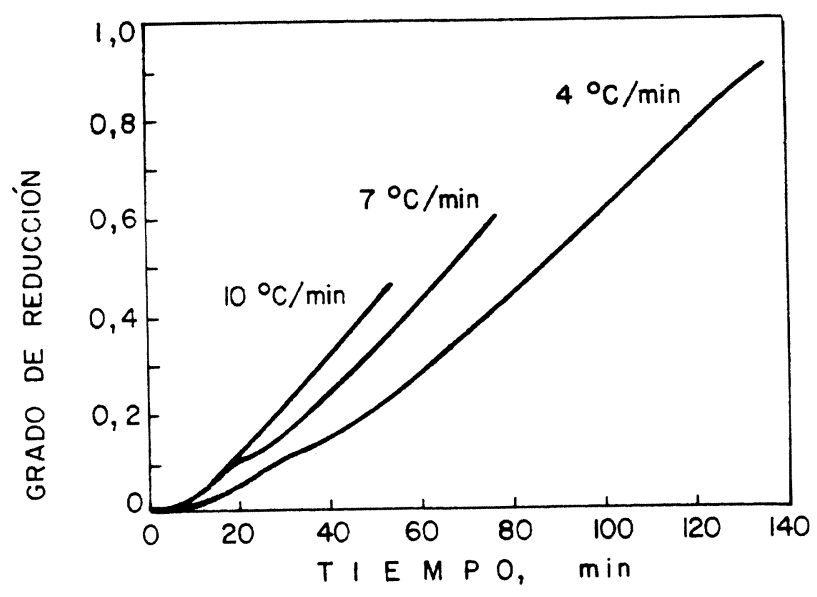

FIg. 1.- Curvas que muestran la tendencia general de la reducción no isotérmica en función de la velocidad de calentamiento. En este caso, pelets de SIDOR reducidos en la mezcla gaseosa $\mathrm{H}_{2}-\mathrm{CO}$.

FIG. 1.- Prevalent trend of nonisothermal reduction in funtion of heating rate. In this case,

SIDOR pellets reduced under $\mathrm{H}_{2}$ - $\mathrm{CO}$ mixture.

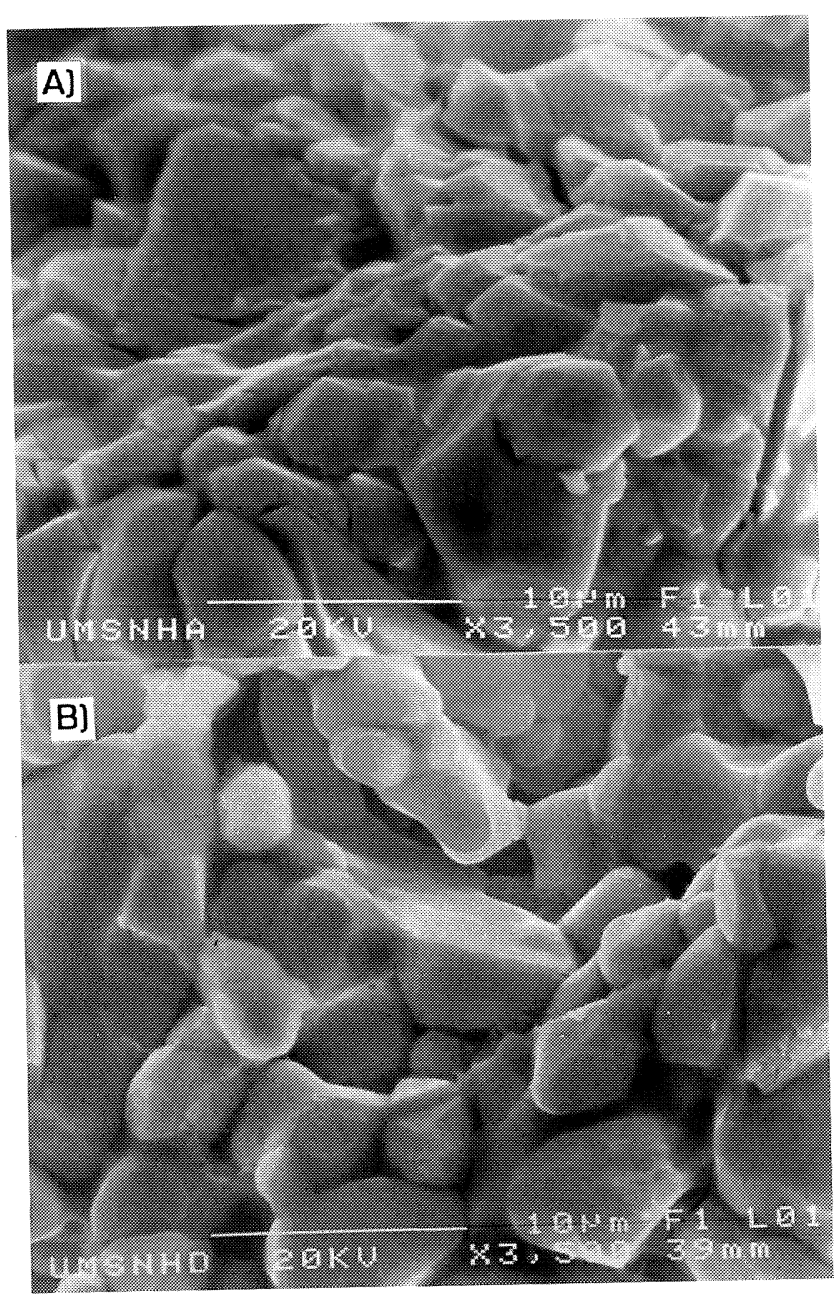

FIG. 2.- Micrografías del pelet de Peña Colorada parcialmente reducido en la mezcla $\mathrm{H}_{2}-\mathrm{CO}$; a) $2,7 \%$ y b) $18 \%$ de reducción.

FIG. 2.- SEM micrographs of Peña Colorada pellets partially reduced in a $\mathrm{H}_{2}-\mathrm{CO}$ mixture;

a) 2,7\% and b) $18 \%$ reduction.

la reducción se observó que a menor velocidad de calentamiento, menor es la temperatura a la que la muestra comienza a perder peso cuando se encuentra en contacto con los gases reductores. Esto se debe a que conforme más lento es el calentamiento, se da mayor oportunidad de respuesta a los cambios que se generan en el sistema de reacción.

La figura 1 muestra el efecto de la velocidad de calentamiento en el proceso de reducción no isotérmico. El eje de abscisas marca como tiempo cero el momento en el cual las diferentes muestras comienzan a reducirse. Se observa que para un mismo tiempo de reacción, el grado de reducción es mayor conforme mayor es la velocidad de calentamiento. Esto se debe a que al incrementar la velocidad de calentamiento, el sistema eleva su temperatura en tiempos más cortos permitiendo mayor avance de la reacción. En el punto final del ciclo de calentamiento no isotérmico $\left(1.000{ }^{\circ} \mathrm{C}\right)$, la reducción es mayor 
en el pelet reducido a menor velocidad de calentamiento ya que permanece expuesto por más tiempo a las temperaturas y gases de reacción.

\subsubsection{Efecto del gas reductor empleado}

La tabla III presenta los valores finales de reducción a las diferentes velocidades de calentamiento para las dos mezclas reductoras empleadas. Se observa que el poder reductor del $\mathrm{H}_{2}$ se manifiesta más para el pelet de Peña Colorada. Sin embargo, en la reducción con $\mathrm{H}_{2}-\mathrm{CO}$ se presume que la mayor área superficial del pelet de SIDOR (Tabla I), induce a que los canales porosos faciliten la llegada de las moléculas de gas $\mathrm{CO}$ a la superficie de las cavidades formadas en el mineral y permitan el avance de la reacción por reducción en estado sólido. En la tabla III se observa que el pelet brasileño de CVDR es ligeramente mejor reducido que el pelet venezolano de SIDOR en la mezcla $\mathrm{H}_{2}$-CO. Esto puede indicar que cuanto mayor sea el área superficial del mineral del que se parte, se induce a una mejor reducción cuando se adiciona $\mathrm{CO}$ como agente reductor a $\mathrm{H}_{2}$ puro.

\subsection{Evaluación de la energía de activación}

La evaluación de los parámetros cinéticos se efectuó por las técnicas propuestas por Coats \& Redfern (1), Dixit \& Ray (2) y Prakash \& Ray (3), con el fin de comparar las tendencias y valores obtenidos por los diferentes métodos. La figura 3 presenta la energía de activación evaluada mediante estas técnicas para la reducción con $\mathrm{H}_{2}$ puro del pelet de Peña Colorada, donde se pueden observar los distintos estados en la reacción de reducción. Se observó que los resultados obtenidos con la técnica de análisis propuesta por Prakash \& Ray son más

TABLA III.- Porcentaje de reducción logrado en condiciones no isotérmicas

TABLE III.- Percent of reduction achieved under nonisothermal conditions

\begin{tabular}{|c|rrrr|}
\hline Mezcla & ${ }^{\circ} \mathrm{C} / \mathrm{min}$ & Peña C. & SIDOR & CVDR \\
\hline & 4 & 92,61 & 85,96 & 94,7 \\
$\mathrm{H}_{2}(100 \%)$ & 7 & 64,08 & 61,89 & 64,02 \\
& 10 & 51,29 & 46,87 & 51,29 \\
\hline & 4 & 90,20 & 91,09 & 94,55 \\
$\mathrm{H}_{2}-\mathrm{CO}(95: 5)$ & 7 & 60,41 & 66,14 & 66,03 \\
& 10 & 45,76 & 50,43 & 50,61 \\
\hline
\end{tabular}
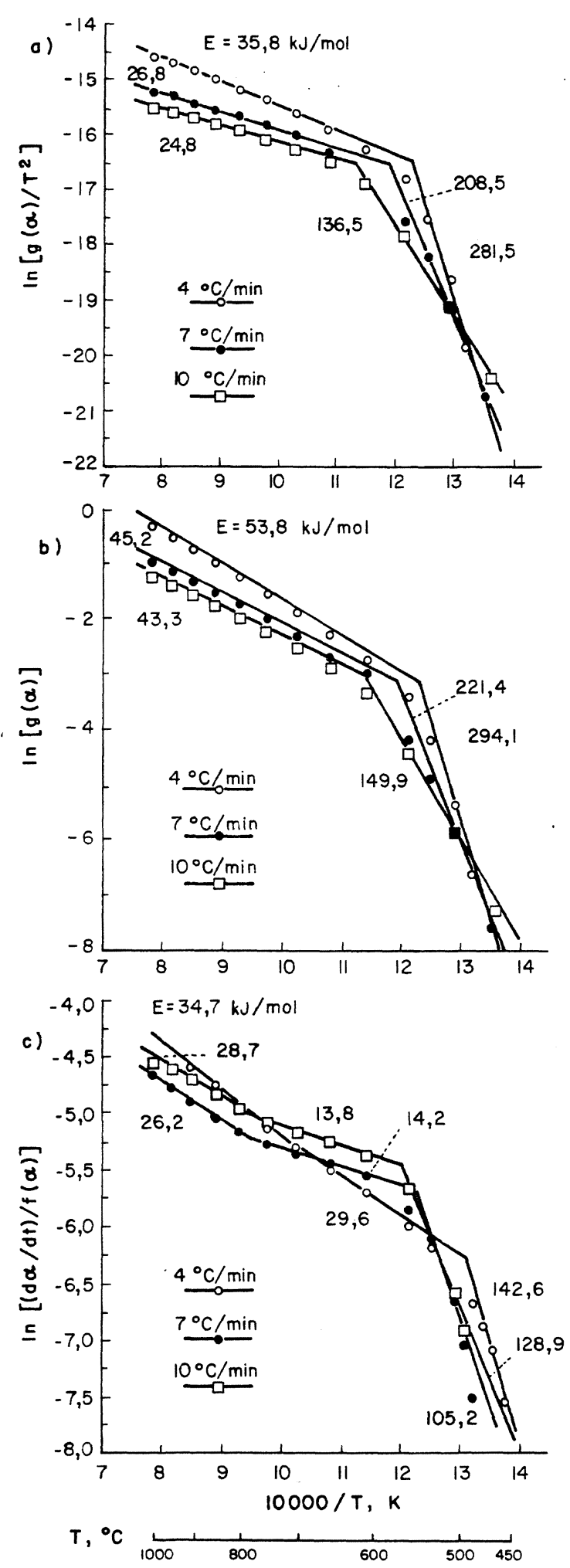

FIG. 3.- Gráficos no isotérmicos de datos TG de acuerdo a: a) Coats \& Redfern, b) Dixit \& Ray y c) Prakash \& Ray, para la reducción del pelet de Peña Colorada con $\mathrm{H}_{2}$ puro.

FIG. 3.- Nonisothermal plots of TG data according to: a) Coats \& Redfern, b) Dixit \& Ray and c) Prakash \& Ray, for reduction of Peña Colorada pellet with pure $\mathrm{H}_{2}$. 
sensibles a los cambios de temperatura, lo que refleja un mayor número de cambios en la pendiente de las curvas a las diferentes velocidades de calentamiento. Sin embargo, a pesar de que los valores individuales calculados por las diferentes técnicas no son iguales, la tendencia de la variación de la energía es semejante independientemente del método de análisis empleado.

\subsubsection{Efecto de la velocidad y gas reductor en la energía de activación}

La región válida de aplicación de los modelos de reacción obtenidos isotérmicamente es de 600 a $1.000{ }^{\circ} \mathrm{C}$. Para hacer un análisis adecuado del efecto del gas reductor y de la velocidad de calentamiento en la reducción no isotérmica en este rango de temperatura, se procedió a usar los resultados obtenidos por el método de Coats \& Redfern con efecto de poder comparar los valores de energía calculados con ayuda de una misma técnica de análisis. Tomando como base lo anterior, la figura 4 muestra el efecto de la velocidad de calentamiento en la energía de activación durante la reducción no isotérmica de los pelets de Peña Colorada, SIDOR, y CVDR reducidos en las mezclas $\mathrm{H}_{2}(100 \%)$ y $\mathrm{H}_{2}$-CO (95:5).

Se observa que la energía de activación disminuye con el incremento de la velocidad de calentamiento. Esto se debe a que cuanto más rápida sea la velocidad de calentamiento, tanto más rápidamente se alcanzan las temperaturas que favorecen la reducción, y disminuye la energía necesaria para que proceda el sistema de reacción. Además, puede considerarse que durante el calentamiento rápido se forma cierta porosidad como resultado de la formación de grietas debido a la gran velocidad de reducción que se alcanza con estas mezclas, lo que produce esfuerzos mecánicos en las partículas como consecuencia del aumento de volumen que resulta al pasar de hematites a magnetita. Esta porosidad formada induce a valores menores de energía de activación. Por el contrario, si la velocidad de calentamiento es lenta, el sistema tarda más tiempo en elevar su temperatura y las necesidades de energía son mayores para que las reacciones sucedan.

Los valores de energía de activación más elevados se obtuvieron para la reducción del pelet de Peña Colorada, probablemente porque presenta menor área superficial en comparación con las otras muestras (Tabla I), lo que disminuye el poder de penetración del agente reductor al interior del pelet y complica el proceso de reducción.

Comparando los valores de energía de activación de las figuras $4 \mathrm{a}$ y $4 \mathrm{~b}$, se observa que la energía es mayor cuando los pelets se reducen con la mezcla $\mathrm{H}_{2}-\mathrm{CO}$ (95:5). Como la velocidad de reducción con $\mathrm{H}_{2}$ puro es mayor que la velocidad de reducción con $\mathrm{CO}$, se puede considerar que la adición de $\mathrm{CO}$ a la mezcla de $\mathrm{H}_{2}$ hace que disminuya la velocidad de reducción de los pelets con esta mezcla, induciendo a un proceso más lento que requiere de mayor energía para llevarse a cabo.

Relacionando los valores de energía de activación calculados de la figura $4 \mathrm{~b}$ para la reducción con la mezcla $\mathrm{H}_{2}-\mathrm{CO}$, respecto del área superficial inicial de los diferentes pelets recogidos en la tabla I, se observa que el área superficial de las muestras influye en la energía de activación resultante. Se comprobó que el orden ascendente de los valores de energía calculados, que son CVDR, SIDOR y Peña Colorada, corresponden a los valores descendentes del área superficial $\left(0,109,0,097\right.$ y $0,072 \mathrm{~m}^{2} / \mathrm{g}$, respectivamente). De esto se concluye que a mayor área superficial de las muestras, corresponden menores necesidades de energía para el proceso de reducción.

\subsubsection{Estudio morfológico}

La figura 5, presenta un montaje de micrografías obtenidas por microscopía óptica donde se ilustran los cambios estructurales que se presentan en la reducción del pelet de Peña Colorada con la mezcla $\mathrm{H}_{2}-\mathrm{CO}$ a diferentes velocidades de calentamiento. Se observa que a menor velocidad de calentamiento, se incrementa la densificación de las muestras y disminuye en gran medida la cantidad y tamaño de los poros. Además, se observa que disminuye la cantidad de óxidos presentes (representados por la fase más obscura) incrementando la cantidad de hierro metálico formado (fase brillante).

Durante el proceso de reducción, los gases de reacción $\mathrm{H}_{2}$ y $\mathrm{CO}$ que penetran en el sólido poroso tienen que adsorberse sobre sitios activos para que, en contacto con los óxidos del pelet pueda llevarse a cabo la reducción. Conforme avanza la reacción, el grado de reducción es mayor y disminuye la cantidad de óxidos presentes $y$, por tanto, disminuye considerablemente la superficie activa sobre la cual pueden adsorberse los gases reactivos. Esto implica que parte de los gases frescos de reacción salgan del interior del pelet sin lograr reaccionar, debido a que la velocidad de difusión de gases a través de la capa porosa es mucho mayor que la capacidad de los mismos para reaccionar y formar productos, como consecuencia del rápido agotamiento de los óxidos. Este mecanismo describe un proceso gobernado por reacción química.

Los modelos por reacción química determinados isotérmicamente para estos procesos cumplen el modelo topoquímico de reducción o del núcleo sin reaccionar, en el cual el frente de reducción avanza desde la periferia hacia el núcleo del pelet. Si las condiciones de reducción así lo permiten, al final 
a) $\mathrm{H} 2(100 \%)$
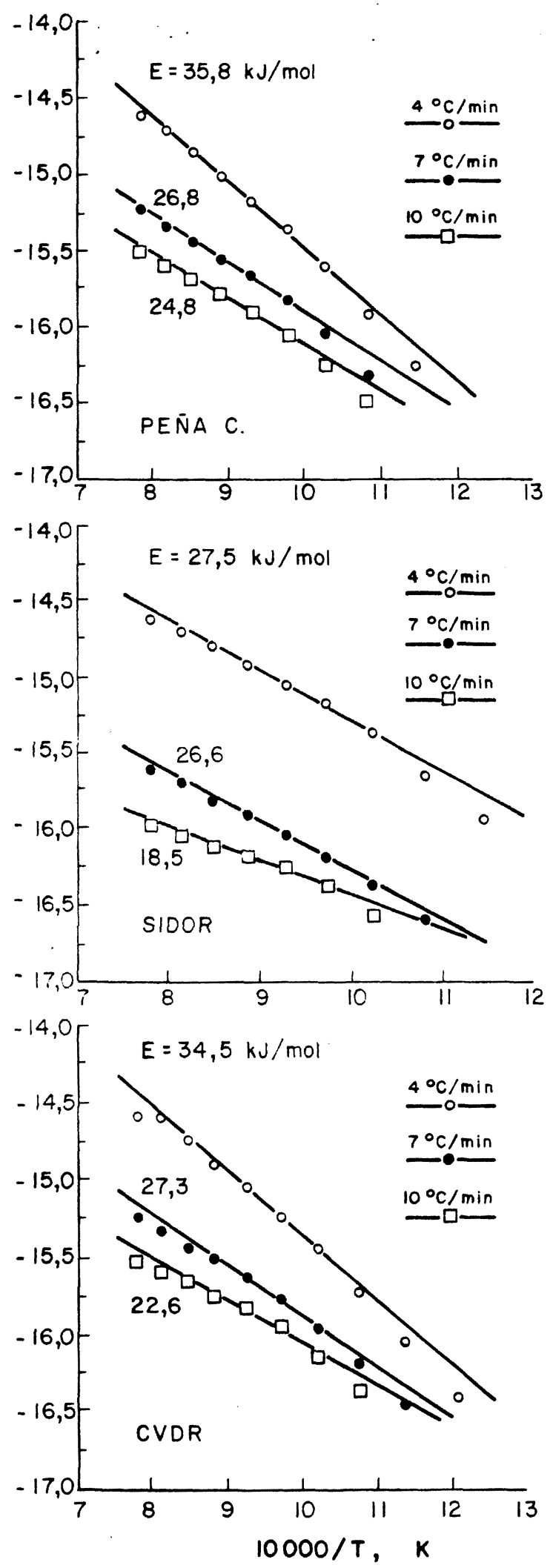

b) $\mathrm{H2}: \mathrm{CO}(95: 5)$
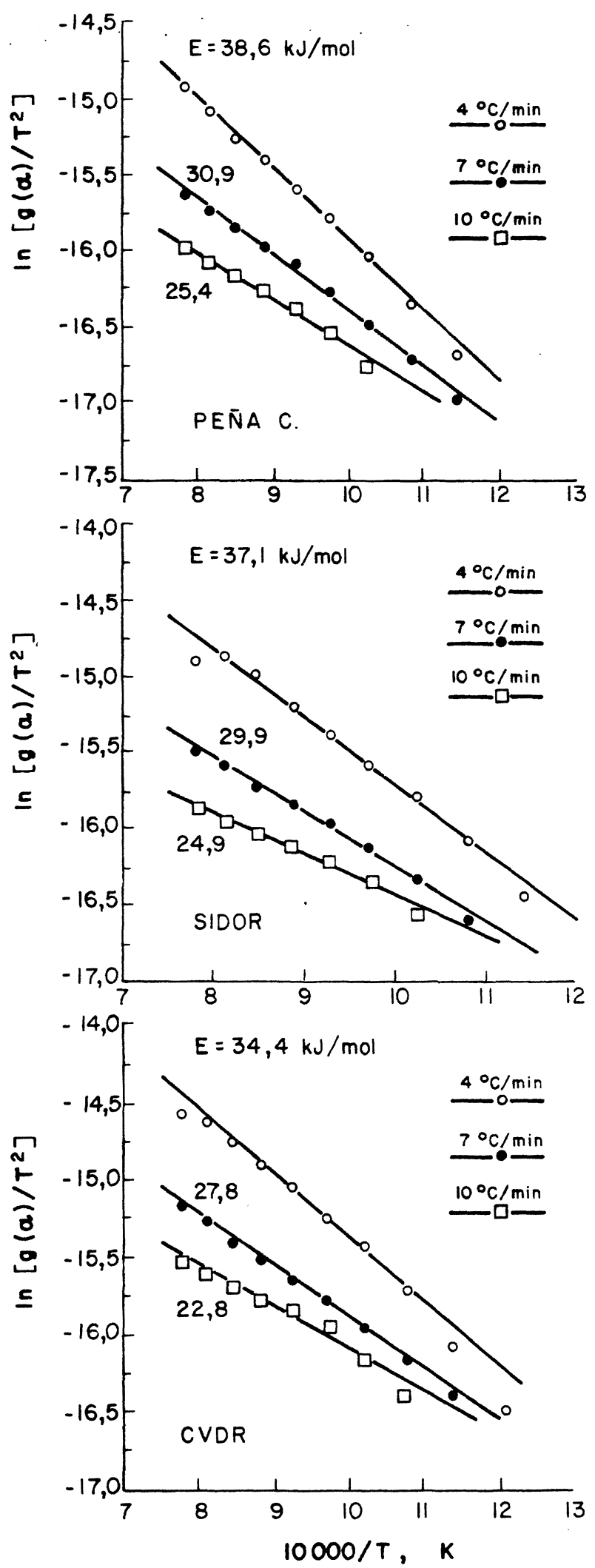

Fig. 4.- Aplicación de la técnica de Coats \& Redfern para los tres pelets empleados; a) $\mathrm{H}_{2}(100 \%) \mathrm{J}$ b) $\mathrm{H}_{2}-\mathrm{CO}(95: 5)$.

FIG. 4.- Coats and Redfern plots for nonisothermal TG data of the three pellets used; a) $\mathrm{H}_{2}$ (100\%) anc b) $\mathrm{H}_{2}-\mathrm{CO}(95: 5)$. 


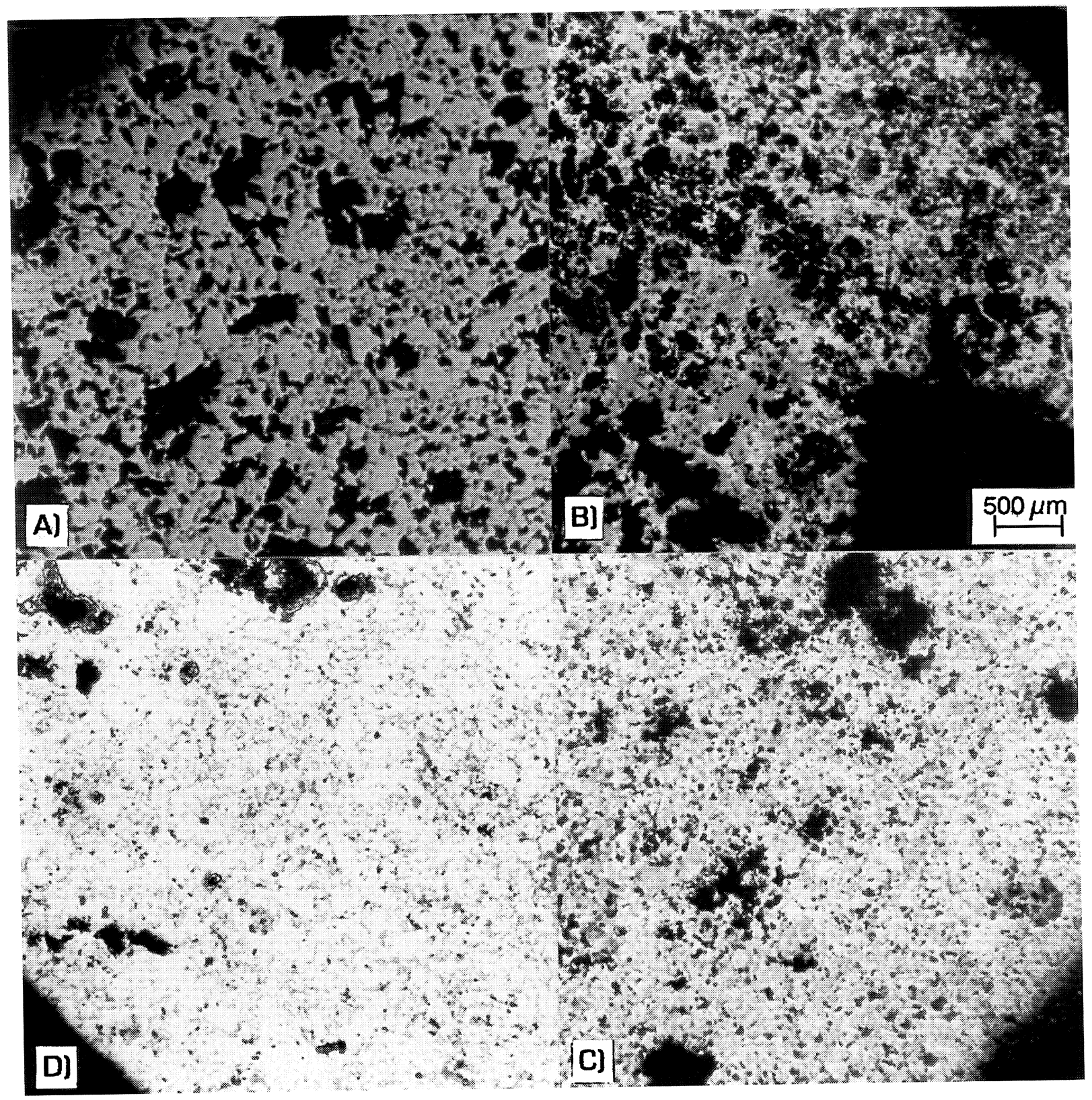

Fig. 5. - Fotomicrografías de secciones pulidas del pélet de Peña Colorada reducido en $\mathrm{H}_{2}$ - $\mathrm{CO}$ a diferente velocidad de calentamiento; a) sin reaccionar, b) $10^{\circ} \mathrm{C} / \mathrm{min}$, c) $7{ }^{\circ} \mathrm{C} / \mathrm{min}$ y d) $4{ }^{\circ} \mathrm{C} / \mathrm{min}$.

FIG. 5.- Photomicrographs of polished sections of Peña Colorada Pellets reduced in $\mathrm{H}_{2}-\mathrm{CO}$ at different heating rates; a) unreacted, b) $10^{\circ} \mathrm{C} / \mathrm{min}$, c) $7{ }^{\circ} \mathrm{C} / \mathrm{min}$ and d) $4{ }^{\circ} \mathrm{C} / \mathrm{min}$.

del proceso se forma una serie de capas concéntricas compuesta por las diferentes fases que van desde hierro metálico hasta su forma más oxidada en el núcleo del pelet.

La figura 6 presenta una micrografía del pelet de Peña Colorada reducido en $\mathrm{H}_{2}$ puro de manera no isotérmica a $7{ }^{\circ} \mathrm{C} / \mathrm{min}$. Esta imagen muestra claramente las características del modelo topoquímico de reducción a nivel pelet. En el borde del pelet se presenta la formación de una capa densa de hierro metálico con porosidad mínima, donde los poros presentes adquieren forma esférica como conse- cuencia de fenómenos de sinterización de la fase hierro. Conforme se avanza al núcleo del pelet, se observa que la porosidad aumenta considerablemente y que los poros son más irregulares y de mayor tamaño. Se observa también que en el centro de la muestra reducida el grado de reducción es menor que en la periferia, y se nota la formación de una interfase entre la fase hierro y los óxidos presentes.

La figura 7a muestra una micrografía por MEB del borde del pelet de SIDOR reducido a $10{ }^{\circ} \mathrm{C} / \mathrm{min}$ en $\mathrm{H}_{2}$ puro. A diferencia de los óxidos de hierro y 


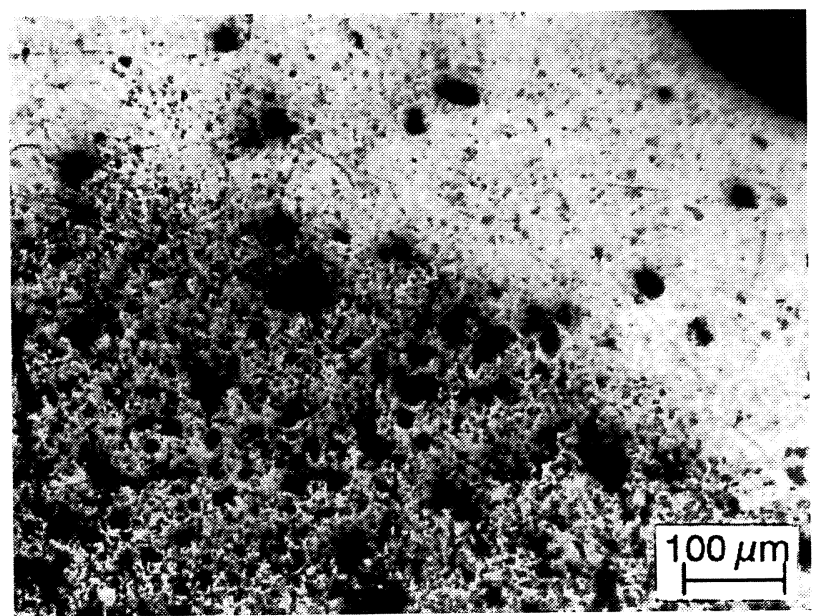

FIG. 6.- Evidencia de reducción topoquímica en una muestra de Peña Colorada reducida a 7 ${ }^{\circ} \mathrm{C} /$ minen $\mathrm{H}_{2}$ puro.

Fig. 6.- Evidence of topochemical reduction in a sample of Peña Colorada reduced at $7{ }^{\circ} \mathrm{C} / \mathrm{min}$.

la gran cantidad de poros presentes en el núcleo del pelet, en el borde de la muestra existe la presencia de hierro metálico con poca porosidad, debido a que esta zona de la muestra se encuentra expuesta de manera más próxima a la acción de los gases reductores. Por el contrario, el núcleo del pelet reaccionó solamente de manera parcial y no se redujo al mismo nivel que la periferia, como consecuencia, en parte, del rápido calentamiento que disminuye el tiempo neto de reacción de los componentes.

La figura $7 \mathrm{~b}$ presenta la morfología del hierro formado cuando se reduce el pelet de SIDOR a 4 ${ }^{\circ} \mathrm{C} / \mathrm{min}$ en la mezcla $\mathrm{H}_{2}$-CO. A esta velocidad de calentamiento menor que la anterior, el pelet permanece expuesto durante más tiempo a las temperaturas elevadas, denotando la presencia de capas densas de hierro debido a fenómenos de sinterización. A diferencia de la reducción con $\mathrm{H}_{2}$ puro, se puede observar la presencia de una gran cantidad de microporos que resultan de la acción reductora del $\mathrm{CO}$ contenido en la mezcla.

Finalmente tomando como base los resultados obtenidos, se puede considerar que el estudio de la cinética de reducción no isotérmica de pelets de mineral de hierro puede constituir un elemento muy importante durante la práctica industrial del proceso de reducción directa, así como para el diseño mismo de los sistemas de reacción, pues se puede obtener información acerca de las propiedades cinéticas y de reducibilidad de los minerales que pueden constituir la materia prima de la carga del reactor, además de la influencia de los diferentes parámetros de operación que interviene un proceso no isotérmico.

La información que se obtiene para un proceso de reducción directa es más realista que la procesa-

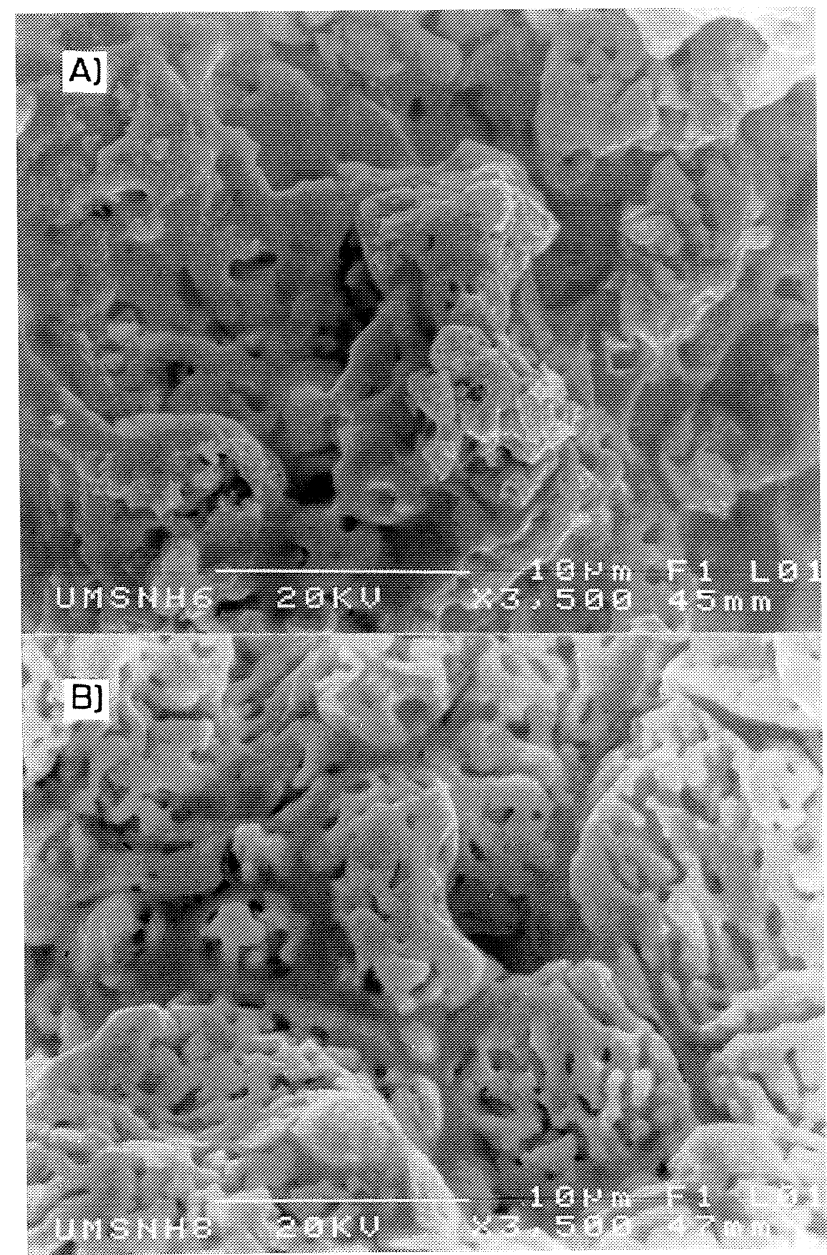

FIG. 7.- Micrografías que muestran la morfología en la periferia del pelet de SIDOR a diferentes condiciones de reducción; a) $10{ }^{\circ} \mathrm{C} / \mathrm{min}, \mathrm{H}_{2}$ puro y b) $4{ }^{\circ} \mathrm{C} / \mathrm{min}, \mathrm{H}_{2}$ - $\mathrm{CO}$.

FIG. 7.- SEM micrographs that show the morphology of the edge of SIDOR pellets reduced at different conditions; a) $10{ }^{\circ} \mathrm{C} / \mathrm{min}$, pure $\mathrm{H}_{2}$ and b) $4{ }^{\circ} \mathrm{C} / \mathrm{min}, \mathrm{H}_{2}$ - $\mathrm{CO}$ mixture.

da por estudio cinético isotérmico tradicional, y en general el estudio cinético no isotérmico puede ser aplicable a un sin número de sistemas heterogéneos que permitan un estudio más adecuado de los diversos procesos industriales actuales.

\section{CONCLUSIONES}

- La reducción isotérmica de los pelets de Peña Colorada en atmósfera de $\mathrm{H}_{2} 100 \%$, así como la de los pelets de CVDR reducidos en $\mathrm{H}_{2}$ puro y en $\mathrm{H}_{2}-\mathrm{CO}$ (95:5), se ajustan al modelo cinético de simetría cilíndrica (CG2).

- La reducción de los pelets de Peña Colorada en la mezcla $\mathrm{H}_{2}-\mathrm{CO}$, así como los pelets de SIDOR reducidos en $\mathrm{H}_{2}$ puro y en $\mathrm{H}_{2}-\mathrm{CO}$ (95:5), 
se ajustan al modelo cinético de reacción de simetría esférica (CG3).

- Los mecanismos de reacción CG2 y CG3 que cumplen el modelo topoquímico de reacción, pueden emplearse en la determinación de los parámetros cinéticos no isotérmicos en el rango de reducción de 600 a $1.000{ }^{\circ} \mathrm{C}$.

- La reducibilidad de las muestras en condiciones no isotérmicas depende de la mezcla empleada. El grado de reducción de los pelets de Peña Colorada se favorece con $\mathrm{H}_{2}$ puro, mientras que para los pelets de SIDOR el grado de reducción es mayor con la mezcla $\mathrm{H}_{2}-\mathrm{CO}$. Los pelets de CVDR no muestran mayor diferencia con ambas mezclas.

- La energía de activación del proceso no isotérmico disminuye con el aumento de la velocidad de calentamiento.

- La energía de activación de los pelets de Peña Colorada es mayor que la de los de SIDOR y CVDR durante la reducción no isotérmica en atmósferas de $\mathrm{H}_{2}$ puro y $\mathrm{H}_{2}-\mathrm{CO}$ (95:5).

- La energía de activación es mayor cuando los pelets son reducidos en la mezcla $\mathrm{H}_{2}-\mathrm{CO}$ que cuando lo son en atmósfera de $\mathrm{H}_{2}$ puro.

- Los valores de energía de activación evaluados en el estudio cinético no isotérmico varían con el método de análisis empleado, sin embargo, son similares tanto la tendencia como la variación de los resultados.

\section{Agradecimientos}

Los autores agradecen a CONACYT y a la Universidad Michoacana de San Nicolás de Hidalgo el apoyo brindado para la realización de este trabajo.

\section{REFERENCIAS}

(1) CoAts, A.W. y RedFern, J.P. Nature, 201, 1964: 68-69.

(2) DiXIT, S.K. y RAY, H.S. Thermochim. Acta, 54, 1982: 245-250.

(3) Prakash, S. y Ray, H.S. ISIJ Int., 30 (3), 1990: 183-191.

(4) Sestak, J. y Berggren, G. Thermochim. Acta, 3, 1971: $1-12$.

(5) Garn, P.D. J. Thermal Anal., (6), 1974: 237-239.

(6) MacCallum, J.R. Nature, 225, 1970: 1.127-1.128.

(7) Felder, R.M. y Stahel, E.P. Nature, 228, 1970: 1.0851.086.

(8) Segal, E. y Vlad, M. Thermochim. Acta, 16, 1976: 115 120.

(9) Sohn, H.Y. y Wadsworth, M.E. Rate Processes of Extractive Metallurgy, Cap. 1, Ed. Plenum Press, Nueva York, 1979: 1-51.

(10) El-Geassy, A.A. y NASR, M.I. Trans. ISIJ, 28, 1988 650-658.

(11) Towhidi, N. y Szekely, J. Metall. Trans., 14B, 1983 359-367.

(12) Ünal, A. y Bradshaw, V. Metall. Trans., 14B, 1983 743-752.

(13) John, D.H., Matthew, S.P. y Hayes, P.C. Metall. Trans., 15B, 1984: 701-708.

(14) PAul, S. y MukherJeE, S. Ironmaking Steelmaking, 19 (3), 1992: 190-193.

(15) PRAKASH, S. Ironmaking Steelmaking, 21 (3), 1994: $237-$ 243.

(16) RAY, H.S. y Kundu, N. Thermochim. Acta, 101, 1986: 107-118.

(17) SARKAR, S.B. y RAY, H.S. Trans. ISIJ, 28, 1988: 1.0061.013.

(18) Bandopadhyay, A., Ganguly, A., Prasad, K.K., SARKAR, S.B. y RAY, H.S. ISIJ Int., 29 (9), 1989: $753-$ 760.

(19) Szekely, J., Evans, J.W. y Sohn, H.Y. Gas Solid Reactions, Cap. 3, Ed, Academic Press, Nueva York, 1976 